\title{
BASE DE COMPARACIÓN DE MALLAS CURRICULARES DE CARRERAS DE INGENIERÍA CIVIL
}

\author{
Mauricio Valle Barra \\ Docente, Pontificia Universidad Católica de Valparaíso, Chile
}

\section{INTRODUCCIÓN}

Los esfuerzos por mejorar la calidad de la educación técnica puede involucrar la modificación de la malla curricular en su estructura y contenidos para responder a los cambios en el perfil de egreso, el cual queda conformado por las exigencias del mercado laboral (Cáceres, 2002). Además, como parte del proceso del diseño curricular, el perfil de ingreso establece las condiciones en conocimientos y actitudes que los estudiantes debieran poseer al momento del ingreso a la carrera. Si los requerimientos de ingreso del estudiante a la carrera evolucionan en la medida que también lo hace el perfil de egreso, entonces la malla curricular deberá adecuarse para optimizar el proceso educativo con el fin de cumplir con los objetivos educativos terminales expresados en el perfil de egreso".

En este contexto, de mejorar la calidad de la educación técnica, es que se promueven iniciativas para modernizar las mallas curriculares. En un principio, la reestructuración de los programas de estudios comienza con la revisión del perfil de ingreso y del perfil de egreso junto con los objetivos (misión) del departamento o unidad académica. El modo de trabajo usual consiste en conformar una comisión encargada de la revisión del currículum. Se lleva a cabo una evaluación del actual programa de estudios a través de encuestas aplicadas a estudiantes, egresados, empleadores y docentes [CNAP]. Posteriormente, se elaboran recomendaciones sobre los cambios tanto en la estructura como en los contenidos del programa. Conlleva también una propuesta de implementación que integre todos los cambios de acuerdo a los resultados de las encuestas y de las revisiones de perfiles y objetivos. Sin embargo, en la práctica, la primera actividad para dicha modernización suele ser la visualización de la actual situación del plan de estudio de la carrera a través de la comparación con otros planes similares (de carreras universitarias similares) de otras universidades. Con esto se puede detectar las principales diferencias (que son tratadas más adelante en este artículo) y falencias o debilidades no detectadas de antemano del plan de estudio.

No es la intención reformar la actual malla curricular para hacerla más parecida a las características propias de otras universidades, sino, conocer realmente las fortalezas y debilidades de la malla curricular actual en función de otras de la competencia. Un estudio comparativo será una fuente adicional de información para efectuar un diseño curricular más acorde a las exigencias actuales y como fundamento para hacer posibles cambios al plan de estudio de la carrera. Además, el ejercicio formal de comparación propuesto en este artículo permite un mecanismo adicional de evaluación del plan de estudio y de los programas de las asignaturas para proponer modificaciones y actualizaciones.

Cuando se menciona que las mallas curriculares a comparar deben ser de carreras similares o afines, se entenderá aquellas carreras que en su módulo contienen la especialidad en cuestión, independiente si los nombres de las carreras difieren en su apellido. Por ejemplo, la carrera de Ingeniería 
Civil Industrial, podría también denominarse en otras universidades Ingeniería Civil de Industrias, o la carrera de Ingeniería Civil Eléctrica podría llamarse Ingeniería Civil Electricista. Para el análisis comparativo, lo importante será que el núcleo de la especialidad sea la misma, independiente del nombre de la misma.

\section{MARCO REFERENCIAL}

Como se ha descrito en la Introducción, la base de comparación está concebida como una actividad que permite al departamento o unidad académica responsable de la carrera universitaria, mejorar la calidad de la enseñanza técnica a través del perfeccionamiento y actualización de la malla curricular de la carrera. El Benchmarking es simplemente medir y comparar el desempeño de procesos o actividades de otras organizaciones del mismo rubro industrial (Stevenson, 1996). El objeto del benchmarking, es aprender de otros sin tener que "reinventar la rueda", usando el conocimiento y experiencia de otros para mejorar. Queda entonces claro, que la base de comparación es una actividad de benchmarking aplicado en el rubro de la educación.

Las tres razones por las cuales el benchmarking es usado en la industria son (Boxwell, 1994):

- El Benchmarking es el modo más rápido y eficiente de efectuar mejoras evitando ensayos y errores (algo crucial en el dominio de los cambios curriculares por el costo en tiempo que eso significa).

- El Benchmarking mejora la habilidad de la organización para acelerar los cambios para las mejoras (a veces la motivación para la mejora y el cambio es mayor cuando las autoridades visualizan cómo la competencia nos está aventajando).

- El Benchmarking tiene la habilidad de elevar el nivel de desempeño general de la industria si todas las instituciones están avocadas en mejorar los estándares mínimos de operación.

Hay tres tipos primarios de benchmarking que se usan. Estos son benchmarking de procesos, de desempeño, y estratégico (Bogan, 1994). La base de comparación de mallas curriculares propuesta cae dentro del tipo de benchmarking de desempeño ya que se enfoca en valorar la posición competitiva a través de la comparación del producto o servicio (en este caso, el plan de estudios de la carrera) con otros competidores. Cuando se trata con este tipo de benchmarking, se desea conocer cómo está el producto o servicio en relación con los de la competencia en cuanto a calidad, confiabilidad, velocidad de entrega y otras características propias del producto o servicio.

El método planteado se enfoca particularmente a la comparación de mallas de carreras de ingeniería civil (en cualquiera de sus disciplinas) cuya duración es de seis años y contempla cinco áreas de formación (según $\mathrm{CNAP}^{2}$ ): Ciencias básicas, Ciencias de la ingeniería, Ingeniería Aplicada, Ciencias sociales y humanidades, y de formación profesional. Lo anterior establece por si misma, una base de comparación, en donde se formulan los porcentajes respecto del total de asignaturas, para cada área de formación. A continuación se detallan las áreas (véase "Criterios de Evaluación para la Carrera de Ingeniería Civil" según CNAP):

\footnotetext{
${ }^{1}$ En general, los cambios en la educación en ingeniería han sido de tipo evolucionarios más que revolucionarios a través del consenso de educadores, el gobierno y los líderes industriales [Tryggvason, 2001].

${ }^{2}$ CNAP: Comisión Nacional de Acreditación de Pregrado (www.cnap.cl)
} 
La primera área de formación es la de 'Ciencias Básicas', correspondiente al tratamiento de las matemáticas, la física, química y otras disciplinas según las características del programa. La función de estas asignaturas es la de contribuir al pensamiento lógico y proporcionar a los estudiantes los fundamentos que les permitirán enfrentarse a los problemas que requieren capacidad analítica en las asignaturas subsiguientes de la especialidad.

La segunda área de formación es la de 'Ciencias de la Ingeniería', correspondiente al tratamiento de las disciplinas relativas a los materiales, las energías, sistemas y procesos con el objeto de entregar bases conceptuales y herramientas de análisis para la ingeniería aplicada. Entre las asignaturas que incluyen esta área están, mecánica de fluidos, termodinámica, dinámica, mecánica, teoría de sistema, computación, programación, etc.

La tercera área de formación es la de 'Ingeniería Aplicada', que incluye los elementos fundamentales del diseño de ingeniería en la especialidad de la carrera en cuestión. Los objetivos de las asignaturas de esta área son la de capacitar al estudiante para la creación y adaptación de tecnologías propias de su área de desempeño y de permitir al estudiante un inicio eficiente en sus servicios profesionales.

La cuarta área de formación es la de 'Ciencias Sociales y Humanidades' con el objeto de lograr una formación integral del profesional, a través del estudio de la sociedad, las relaciones individuales con ella y también aspectos de índole económico-financiero que potencien al profesional en áreas no atingentes a la propia.

Finalmente, la quinta área de formación es la de 'Electivos de formación Profesional' que complementa y/o profundiza en la formación profesional del estudiante en materias que no están explícitamente incluidas como obligatorias en el plan de la carrera pero que se deben cursar y pueden tener relación con cualquier área de las mencionadas anteriormente.

\section{MÉTODO DE COMPARACIÓN}

La comparación de las mallas curriculares está basada en el análisis de las diferencias en asignaturas existentes en las mallas, determinando el esfuerzo porcentual (número de asignaturas sobre el total) aplicado en las áreas de formación que una carrera de ingeniería civil debe considerar según la CNAP, y que se dividen en: Ciencias básicas, Ciencias de la Ingeniería, Ingeniería Aplicada, Ciencias Sociales y Humanidades y Electivos de formación profesional.

No se pretende establecer comparaciones en términos de la concordancia del plan de estudio con la(s) definición(es) del perfil de egreso y los objetivos de cada unidad responsable de la carrera de interés, ya que esa tarea es propia de cada unidad y se escapa a los objetivos de este trabajo. Tampoco se pretende efectuar comparaciones en cuanto al contenido de los programas de asignaturas. Los criterios de evaluación y los métodos pedagógicos tampoco son parte del análisis comparativo ${ }^{3} \mathrm{o}$ de benchmarking.

Un aspecto que se debe considerar en la comparación como base para producir cambios futuros al plan de estudios, es la flexibilidad que éste contiene, en la manera de acercar la estructura curricular a una

\footnotetext{
${ }^{3}$ En un contexto más amplio del currículo de la carrera, se incluye el conjunto de objetivos, contenidos, métodos pedagógicos y criterios de evaluación en cada una de los niveles, etapas, o ciclos, sin embargo, aquí sólo se desea evaluar comparativamente el currículo de la carrera en términos de las asignaturas que la conforman.
} 
más integrada, en la que el alumno adquiera habilidades específicas de la especialidad de la ingeniería desde temprano. La comparación de la flexibilidad a la que se alude, se mide en términos de cuántas asignaturas de la especialidad (Ingeniería aplicada y en Ciencias de la ingeniería) se consagran en los primeros cuatro semestres de la carrera. Además se incluye el porcentaje de las asignaturas respecto del total que son de tipo electivas en las áreas de la especialidad, como elemento de medición de la flexibilidad del plan de estudios.

A continuación se detallan los distintos tipos de comparaciones:

\subsection{Comparación por áreas de formación}

La comparación por áreas de formación consiste en determinar el porcentaje de asignaturas respecto del total de asignaturas de la carrera, para cada una de las áreas de formación descritas en el marco referencial. De esta forma se puede visualizar rápidamente el esfuerzo que cada plan de estudio le dedica a cada área.

En general, se ha constatado (en estudios informales realizados por el autor de este artículo) que en carreras de ingeniería civil, se conforma un patrón de porcentajes similar en carreras de la misma especialidad.

\subsection{Comparación por tipo de actividad}

La comparación por tipo de actividad, divide las asignaturas del plan de estudio en asignaturas teóricas, prácticas, de formación ética y de proyecto de título. El número de asignaturas de cada actividad se expresa en porcentaje sobre el total de asignaturas del plan de estudios.

Respecto a las actividades prácticas, se entenderán aquellas que hagan uso de infraestructura de laboratorio, en donde se ponga en práctica la teoría adquirida en las asignaturas teóricas cursadas con anterioridad o en paralelo a la actividad práctica mediante instrumentos de medición, máquinas o softwares. Las asignaturas de proyecto de título hacen mención al trabajo final que el estudiante realiza para optar al título de ingeniero, que por lo general tiene una duración de un año académico para ingeniería civil (dos semestres), equivalente a dos asignaturas.

\subsection{Comparación de la flexibilidad}

La comparación de las flexibilidades de los planes curriculares se entiende en este estudio como la capacidad que tiene el plan de asumir asignaturas de la especialidad (ingeniería aplicada) o de ciencias de la ingeniería en momentos tempranos al inicio de la carrera. Al inicio de la carrera se entenderá en alguno de los primeros cuatro semestres del plan de estudio. Los primeros cuatro semestres de la carrera, equivalen a los dos primeros años de contacto del estudiante con su elección profesional, por tal motivo, se ha elegido ese período de tiempo para verificar cuál es el grado de acercamiento que la carrera ofrece a sus nuevos estudiantes.

Mientras mayor el número de asignaturas de las áreas de ciencias de la ingeniería y de ingeniería aplicada al inicio de la carrera, se entenderá que el plan de estudio tiene mayor grado de flexibilidad.

Además, se incluye en el concepto de flexibilidad, el grado de posibilidad que el estudiante tenga de poder especializarse, eligiendo asignaturas de su preferencia en áreas propias de su carrera dentro de una gama amplia de opciones. De esta forma, el alumno puede -con ciertas limitaciones-, conformar su propio 
plan de estudios de acuerdo a sus propias preferencias. Estas asignaturas son de tipo electivo y obligatorios. Mientras mayor sea el porcentaje de este tipo de asignaturas, se entenderá que el plan de estudio tiene mayor grado de flexibilidad.

\subsection{Comparación Excluyente}

La comparación excluyente de asignaturas permite verificar qué asignaturas tiene y no tiene un plan de estudios en particular respecto a otros planes de otras universidades con carreras similares (de la misma especialidad). Este análisis se efectúa agrupando las asignaturas por áreas de formación.

Para verificar "qué" asignaturas tiene el plan de estudio a analizar, se fabrica una tabla en la cual se van registrando todas las asignaturas que son comunes a ambos planes (en análisis y el de comparación).

Para verificar que asignaturas "no" tiene el plan de estudio a analizar, se fabrica también una tabla en la que se van registrando las asignaturas que tiene el plan de estudio en análisis y que no están considerados en el plan de estudio de comparación. Es la actividad inversa a la descrita inicialmente; en una se verifica qué asignaturas no están en plan de estudio y que los otros planes si las tienen, y en la otra se verifica qué asignaturas incluye en plan de estudio y que los otros planes no las tienen.

Los ejercicios anteriores son útiles para descubrir una posible debilidad del plan de estudio por obsolescencia de contenidos de asignaturas que ya debieran ser descontinuadas o modificadas para adaptarlas a la realidad tecnológica actual. Esto es de especial consideración en carreras técnicas en vista de los rápidos avances tecnológicos que producen nuevas tecnologías, aumentando la posibilidad de que el plan de estudio de dichas carreras sufra de obsolescencia, de modo que es necesario una revisión periódica de las asignaturas y sus contenidos para mantenerla actualizada.

\section{RESULTADOS}

Se ha aplicado el método de comparación descrito para el caso particular de las carreras de ingeniería civil en la disciplina de Electricidad y Electrónica de cinco universidades tradicionales de la zona central del país. En la Tabla 1 se tienen los datos de las Universidades consideradas con los respectivos nombres de las carreras en análisis.

Se han elegido las carreras mencionadas en el Tabla 1 puesto que todas pertenecen a Universidades públicas con una larga trayectoria y tradición en el desarrollo de carreras de ingeniería.

Tabla 1

\begin{tabular}{|l|l|}
\hline Universidad & Nombre de la Carrera \\
\hline Pontificia Universidad Católica de Valparaíso & Ingeniería Civil Electrónica \\
\hline Pontificia Universidad Católica de Chile & Ingeniería Civil Electricista \\
\hline Universidad Técnica Federico Santa María & Ingeniería Civil Electrónica \\
\hline Universidad de Chile & Ingeniería Civil Electricista \\
\hline Universidad de Santiago de Chile & Ingeniería Civil en Electricidad \\
\hline
\end{tabular}

Los datos provienen de la información pública de las mallas curriculares disponibles en los sitios web de cada universidad (ver referencias). 


\subsection{Comparación por áreas de formación}

Las áreas de formación han sido descritas en la Introducción y se usarán como elementos de comparación para visualizar la estructura de las mallas curriculares. En la Tabla 2 se aprecian los resultados. Nótese que el análisis está efectuado por asignaturas y porcentajes de asignaturas respecto del total que cada plan contiene.

Tabla 2

Resultados de la comparación de los planes de estudio por áreas de formación

\begin{tabular}{|r|cc|c|c|c|}
\hline & \multicolumn{5}{|c|}{ Porcentaje respecto del total de asignaturas } \\
\hline Área de formación & $\begin{array}{c}(1) \\
\text { Total de } \\
\text { asignaturas: } \\
\end{array}$ & $\begin{array}{c}(2) \\
\text { Total de } \\
\text { asignaturas: } \\
53\end{array}$ & $\begin{array}{c}(3) \\
\text { Total de } \\
\text { asignaturas: } \\
57\end{array}$ & $\begin{array}{c}(4) \\
\text { Total de } \\
\text { asignaturas: } \\
61\end{array}$ & $\begin{array}{c}\text { Total de } \\
\text { asignaturas: } \\
58\end{array}$ \\
\hline Ciencias básicas & $22,3 \%$ & $22,2 \%$ & $24,6 \%$ & $19,7 \%$ & $27,6 \%$ \\
\hline Ciencias de la ingeniería & $12,7 \%$ & $11,2 \%$ & $12,3 \%$ & $4,9 \%$ & $8,6 \%$ \\
\hline Ingeniería aplicada & $36,5 \%$ & $35,2 \%$ & $28,0 \%$ & $32,8 \%$ & $31,1 \%$ \\
\hline Ciencias sociales y humanidades & $9,5 \%$ & $14,8 \%$ & $3,5 \%$ & $18,0 \%$ & $3,4 \%$ \\
\hline Electivos profesionales & $19,0 \%$ & $16,6 \%$ & $31,6 \%$ & $24,6 \%$ & $29,3 \%$ \\
\hline Total & $100 \%$ & $100 \%$ & $100 \%$ & $100 \%$ & $100 \%$ \\
\hline
\end{tabular}

(1) Pontificia Universidad Católica de Valparaíso

(2) Universidad de Santiago de Chile

(3) Pontificia Universidad Católica de Chile

(4) Universidad Técnica Federico Santa María (no se consideran asignaturas de deporte)

(5) Universidad de Chile

Se puede constatar que la distribución del esfuerzo por área de formación en las carreras de ingeniería civil con especialidad en electrónica y electricidad, es de 23,3\% para Ciencias básicas, 9,9\% para Ciencias de la ingeniería, 32,7\% para Ingeniería aplicada, 9,8\% para Ciencias sociales y humanidades, y un $24,3 \%$ para Electivos profesionales. Los porcentajes anteriores corresponden al promedio de los porcentajes individuales para cada carrera. Bajo esta información, se podría poner bajo análisis, un plan de estudio de una de las cinco universidades y someterla a una comparación entre su distribución de porcentajes y las del promedio. Por ejemplo, el plan de estudios de la Pontificia Universidad Católica de Chile, tiene comparativamente menos cantidad de asignaturas en el área de Ciencias sociales y humanidades que el resto de los planes de estudio; lo mismo ocurre con Electivos profesionales. Sin embargo, en Ingeniería Aplicada tiene una mayor cantidad de asignaturas de este tipo que los demás planes.

\subsection{Comparación por tipos de actividades}

En la Tabla 3 se presentan los porcentajes de las asignaturas respecto del total del plan de estudio de cada carrera según el área de actividad.

Tabla 3

Resultados de la comparación de los planes de estudio por áreas de actividad

\begin{tabular}{|r|c|c|c|c|c|}
\hline & \multicolumn{5}{|c|}{ Porcentaje respecto del total de asignaturas } \\
\hline Tipo de actividad & $(1)$ & $(2)$ & $(3)$ & $(4)$ & $(5)$ \\
\hline Actividades teóricas & $84,1 \%$ & $83,3 \%$ & $75,5 \%$ & $80,3 \%$ & $84,4 \%$ \\
\hline Actividades prácticas & $9,5 \%$ & $9,3 \%$ & $10,5 \%$ & $9,8 \%$ & $8,6 \%$ \\
\hline Formación ética & $3,4 \%$ & $3,7 \%$ & $14,0 \%$ & $6,6 \%$ & $3,5 \%$ \\
\hline Tesis/proyecto & $3,4 \%$ & $3,7 \%$ & - & $3,3 \%$ & $3,5 \%$ \\
\hline Total & $100 \%$ & $100 \%$ & $100 \%$ & $100 \%$ & $100 \%$ \\
\hline
\end{tabular}

(1) Pontificia Universidad Católica de Valparaíso

(2) Universidad de Santiago de Chile 
(3) Pontificia Universidad Católica de Chile

(4) Universidad Técnica Federico Santa María (no se consideran asignaturas de deporte)

(5) Universidad de Chile

La Pontificia Universidad Católica de Chile es la que presenta la mayor cantidad de actividades prácticas. Con las demás actividades, tanto teóricas como de formación ética y de tesis/proyecto, se presenta similitud en sus porcentajes de actividad, aunque nuevamente, la Pontificia Universidad Católica de Chile destaca por la menor cantidad de asignaturas teóricas en vista de la mayor cantidad de asignaturas dedicadas a la formación ética. En actividades de tesis y proyecto, todos los planes dedican la misma cantidad de asignaturas (que serían dos), excepto la Pontificia Universidad Católica de Chile que tiene estas actividades fuera de su estructura curricular, aunque también de carácter obligatorio para sus estudiantes.

Al igual que en la comparación por áreas de formación, se establece un patrón de distribución de porcentajes de actividades para este tipo de especialidad de la ingeniería formada por el promedio de los porcentajes individuales por área que sería el siguiente: $81,5 \%$ en actividades teóricas, 9,5\% en actividades prácticas, $6,2 \%$ en formación ética y de 3,5\% en Tesis/proyecto.

\subsection{Comparación de las flexibilidades}

En la Tabla 4 se presenta los resultados que se deben interpretar de la siguiente forma: de la tercera a la sexta fila se encuentran la cantidad de asignaturas en el área de ciencias de la ingeniería y de ingeniería aplicada que están disponibles en alguno de los primeros cuatro semestres del plan de estudio ordenadas según universidad. En la última fila se encuentra el porcentaje de asignaturas de carácter electivo de "especialización" o "de carrera" respecto del total de asignaturas.

Tabla 4

Resultados de la comparación de los planes de estudio en cuanto a flexibilidad

\begin{tabular}{|c|c|c|c|c|c|c|c|c|c|c|}
\hline Ues. & \multicolumn{2}{|c|}{ (1) } & \multicolumn{2}{|c|}{ (2) } & \multicolumn{2}{|c|}{ (3) } & \multicolumn{2}{|c|}{ (4) } & \multicolumn{2}{|c|}{ (5) } \\
\hline Semestre & (a) & (b) & (a) & (b) & (a) & (b) & (a) & (b) & (a) & (b) \\
\hline Primero & 0 & 1 & 1 & 0 & 0 & 0 & 1 & 0 & $\mathrm{~s} / \mathrm{i}$ & $\mathrm{s} / \mathrm{i}$ \\
\hline Segundo & 2 & 0 & 0 & 1 & 0 & 0 & 1 & 0 & $\mathrm{~s} / \mathrm{i}$ & $\mathrm{s} / \mathrm{i}$ \\
\hline Tercero & 0 & 0 & 0 & 0 & 0 & 1 & 0 & 1 & $\mathrm{~s} / \mathrm{i}$ & $\mathrm{s} / \mathrm{i}$ \\
\hline Cuarto & 1 & 2 & 1 & 2 & 1 & 2 & 0 & 4 & $\mathrm{~s} / \mathrm{i}$ & $\mathrm{s} / \mathrm{i}$ \\
\hline Total & 3 & 2 & 2 & 3 & 1 & 3 & 2 & 5 & $\mathrm{~s} / \mathrm{i}$ & $\mathrm{s} / \mathrm{i}$ \\
\hline $\begin{array}{r}\text { porcentaje } \\
\text { electivos }\end{array}$ & \multicolumn{2}{|c|}{$9,5 \%$} & \multicolumn{2}{|c|}{$16,6 \%$} & \multicolumn{2}{|c|}{$17,5 \%$} & \multicolumn{2}{|c|}{$11,4 \%$} & \multicolumn{2}{|c|}{$17,2 \%$} \\
\hline
\end{tabular}

(1) Pontificia Universidad Católica de Valparaíso

(2) Universidad de Santiago de Chile

(3) Pontificia Universidad Católica de Chile

(4) Universidad Técnica Federico Santa María (no se consideran asignaturas de deporte)

(5) Universidad de Chile

(a) Asignaturas del área Ciencias de la Ingeniería

(b) Asignaturas del área de Ingeniería Aplicada

Nota: La última fila hace referencia a las asignaturas de carácter electivo (usualmente para adquirir especialización) pero no opcionales, es decir, son asignaturas que se deben cursar de una gama de opciones según la elección del estudiante y que corresponden a materias propias del área de "Ingeniería Aplicada".

Se aprecia el plan de la carrera de la Pontificia Universidad Católica de Valparaíso es la menos flexible en cuanto a la posibilidad de asignaturas electivas para acceder a especialización con sólo un 9,5\%, también la Universidad Santa María presenta cierto grado de rigidez al respecto con un $11,4 \%$. Los planes que más destacan son los de la Pontificia Universidad Católica de Chile, la Universidad de Santiago y la Universidad de Chile que presentan un porcentaje superior al 16\% en asignaturas de libre elección de ingeniería aplicada. En estos casos, se optan por título con menciones en algún área en particular. Sin 
embargo, al realizar una comparación con la capacidad que tienen los planes de aceptar asignaturas de la especialidad en los primeros semestres de la carrera, la situación se revierte, siendo las universidades con mayor grado de opción de especialización las que menor cantidad de asignaturas de ingeniería aplicada o de ciencias de la ingeniería tienen en algunos de los primeros cuatro semestres. Aquí, el plan de la carrera de la Pontificia Universidad Católica de Valparaíso destaca con 5 asignaturas junto con el de la Universidad de Santiago y la Universidad Santa María con 7 asignaturas. Respecto a la Universidad de Chile, no se tiene información de la ubicación de las asignaturas en los semestres correspondientes.

\subsection{Comparación excluyente de asignaturas}

Para realizar este tipo de comparación, se debe elegir un plan de estudios base con el cual comparar. Para este propósito se considerará el plan de estudio de la carrera de Ingeniería Civil Electrónica de la Pontificia Universidad Católica de Valparaíso (PUCV).

En la Tabla 5 se presenta un resumen de las asignaturas que otras universidades tienen como obligatorias en su plan de estudios y que el plan de la carrera de la PUCV no las contempla.

\section{Tabla 5}

Asignaturas existentes en otros planes de carrera ordenados según universidad que no están contempladas en el plan de estudio de la carrera de Ingeniería Civil Electrónica de la PUCV.

\begin{tabular}{|c|c|c|c|c|}
\hline Área & (2) & (3) & (4) & (5) \\
\hline Ciencias Básicas & & Química general. & Química y sociedad. & $\begin{array}{l}\text { Probabilidades y } \\
\text { procesos } \\
\text { estocásticos. } \\
\text { Química moderna. }\end{array}$ \\
\hline $\begin{array}{r}\text { Ciencias de la } \\
\text { Ingeniería }\end{array}$ & $\begin{array}{l}\text { Sistemas de } \\
\text { computación. } \\
\text { Laboratorio de } \\
\text { computación. }\end{array}$ & $\begin{array}{l}\text { Modelos } \\
\text { estocásticos. } \\
\text { programación } \\
\text { matemática. }\end{array}$ & $\begin{array}{l}\text { Probabilidades y } \\
\text { procesos aleatorios. }\end{array}$ & $\begin{array}{l}\text { Computación. } \\
\text { Sistemas dinámicos. } \\
\text { Seminario de } \\
\text { diseño. }\end{array}$ \\
\hline Ingeniería Aplicada & $\begin{array}{l}\text { Laboratorio de } \\
\text { control y } \\
\text { microcomputadores. }\end{array}$ & $\begin{array}{l}\text { Laboratorio de } \\
\text { telecomunicaciones. } \\
\text { Laboratorio de } \\
\text { control automático. }\end{array}$ & $\begin{array}{l}\text { Proyectos } \\
\text { electrónicos. } \\
\text { Laboratorio de } \\
\text { comunicaciones. } \\
\text { Laboratorio de } \\
\text { control automático. } \\
\text { Sistemas } \\
\text { electromecánicos }\end{array}$ & $\begin{array}{l}\text { Análisis y } \\
\text { modelación de } \\
\text { sistemas dinámicos. } \\
\text { Procesamiento } \\
\text { digital de la } \\
\text { información. } \\
\text { Sistemas para el } \\
\text { procesamiento de la } \\
\text { información. } \\
\text { Sistemas eléctricos } \\
\text { de potencia. }\end{array}$ \\
\hline $\begin{array}{r}\text { Ciencias sociales y } \\
\text { humanidades }\end{array}$ & $\begin{array}{l}\text { Finanzas. } \\
\text { Antropología e } \\
\text { ingeniería. } \\
\text { Comportamiento } \\
\text { humano en el } \\
\text { trabajo. } \\
\text { Evaluación de } \\
\text { proyectos. } \\
\text { Administración de } \\
\text { empresas. } \\
\text { Dirección y gestión } \\
\text { de empresas. }\end{array}$ & Microeconomía. & $\begin{array}{l}\text { Información y } \\
\text { control financiero. } \\
\text { Administración } \\
\text { general. } \\
\text { Inglés. }\end{array}$ & $\begin{array}{l}\text { Evaluación de } \\
\text { proyectos. } \\
\text { Inglés. }\end{array}$ \\
\hline
\end{tabular}

(2) Universidad de Santiago de Chile

(3) Pontificia Universidad Católica de Chile

(4) Universidad Técnica Federico Santa María (no se consideran asignaturas de deporte)

(5) Universidad de Chile

Nota: Las asignaturas que se incluyen en la tabla son parte de las obligatorias para todos los estudiantes de la carrera. No se han incluido asignaturas de especializaciones para optar a una mención. 
Se aprecia que en asignaturas de ciencias básicas, el plan de carrera de la PUCV no considera las ciencias de la química mientras que tres de cuatro universidades sí las tienen. En ciencias de la ingeniería se destaca la ausencia de laboratorio de computación en el plan de la carrera de la PUCV y también asignaturas relacionadas con procesos aleatorios y estocásticos. En ingeniería aplicada, sobresalen los laboratorios de telecomunicaciones, de control automático y de microcomputadores además de una asignatura de proyectos electrónicos. Aunque la malla curricular de la PUCV contempla un 9,5\% de asignaturas de ciencias sociales y humanidades (incluye asignaturas de economía), existen varios como Inglés, evaluación de proyectos, y administración de empresas que no están contemplados en ella. También se agregan microeconomía, dirección y gestión de empresas entre otros.

Tabla 6

Asignaturas existentes en el plan de estudios actual de la carrera de al ElE-electrónica que no están incluidos en ningún plan de otra universidad

\begin{tabular}{|l|l|}
\hline Área & Asignaturas \\
\hline Ciencias Básicas & - \\
\hline Ciencias de la Ingeniería & Resistencia de materiales \\
& Tecnología de los materiales \\
& Dibujo de ingeniería \\
\hline Ingeniería Aplicada & Electrónica de estado sólido \\
& Circuitos electrónicos avanzados \\
& Circuitos electrónicos digitales \\
\hline Ciencias Sociales y humanidades & Contabilidad \\
\hline
\end{tabular}

En la Tabla 6 se efectúa el análisis contrario del anterior, es decir, se mencionan aquellas asignaturas que contempla el actual plan de carrera de electrónica de la PUCV que no están considerados en "ningún" otro plan de otra universidad. En este caso, destacan asignaturas de ciencias de la ingeniería como Resistencia de materiales, Tecnología de los materiales y dibujo de ingeniería. En ingeniería aplicada, no existen asignaturas de un semestre de duración dedicadas a tratar exclusivamente tópicos de electrónica del estado sólido; tampoco se detecta en los programas, dos asignaturas por separadas para tratar tópicos de circuitos electrónicos avanzados y circuitos electrónicos digitales. Es posible que esto último sea un indicio de un enfoque algo obsoleto en del plan de estudio que será necesario modificar. En el área de ciencias sociales y humanidades, sólo destaca la asignatura de contabilidad, aunque se pone de manifiesto que otros planes de estudio incluyen una asignatura de finanzas.

\section{CONCLUSIONES}

El análisis comparativo realizado permite constatar los elementos diferenciadores que cada plan curricular posee respecto a los demás. Desde un punto de vista estratégico, estos elementos diferenciadores caen dentro de la categoría de fortalezas o debilidades, las cuales, como se mencionaron en la Introducción, deberán corregirse, en el caso de debilidades, o potenciarse, en el caso de fortalezas. Esta motivación al cambio es la que se enmarca en el contexto de mejorar la calidad de la educación técnica a través del replanteamiento de la actual malla curricular de la carrera de Ingeniería Civil.

En el caso particular analizado, para el plan de estudios de Ingeniería Civil Electrónica de la Pontificia Universidad Católica de Valparaíso (PUCV), se identifica la escasa flexibilidad que posee el plan de estudio para otorgar a los estudiantes diferentes opciones de especialización. Aunque el actual plan de estudio contempla asignaturas optativas, en comparación con las demás universidades, son pocas las 
asignaturas electivas de tipo de 'Ingeniería Aplicada' de especialización, que el estudiante tiene a disposición.

Cabe destacar, que aunque el plan de estudio de la carrera de la PUCV posee menos flexibilidad en cuanto a asignaturas optativas y electivas, se distingue por estar en el segundo lugar en tener asignaturas de ciencias de la ingeniería e Ingeniería aplicada en los primeros cuatro semestres de la carrera, seguido de la Universidad Santa María.

También se evidencia la existencia de asignaturas en el plan actual de la carrera de la PUCV que no se incluyen en las mallas de otras universidades como es el caso de resistencia de materiales, dibujo de ingeniería, electrónica del estado sólido, dos asignaturas de circuitos electrónicos y contabilidad. Además, existen asignaturas que otras universidades si las tienen incluidas en sus planes de estudios y que el plan actual de estudios de la PUCV no los tiene, como es el caso de procesos estocásticos y procesos aleatorios, laboratorio de telecomunicaciones, laboratorio de control automático y microcomputadores, inglés y evaluación de proyectos.

Resultados cualitativos como los expresados en los párrafos anteriores, aportan información adicional y relevante, en diferentes dimensiones, para efectuar posteriores cambios al actual plan de estudios.

El modelo de comparación de planes de estudio explicado en este artículo, se ha enfocado a determinar elementos diferenciadores entre planes de estudios de Ingeniería Civil de universidades públicas, sin embargo, el autor no sospecha algún inconveniente para aplicar la base de comparación para carreras de ingeniería civil de universidades privadas.

Por la simplicidad en ejecución de las comparaciones y de los análisis los datos extraídos de las mallas curriculares, sería posible utilizar la base de comparación para realizar un análisis de situación actual general de las carreras de ingeniería civil, aplicada a todas las especialidades y conformar así, un grupo de resultados comparativos a nivel nacional, por especialidad y por época, lográndose dilucidar las tendencias de diseño curricular, afectas a las condiciones externas del mercado y de las nuevas tecnologías de enseñanza-aprendizaje.

Una interesante extensión de la base de comparación propuesta, resulta en su aplicación en carreras no ingenieriles, como por ejemplo contabilidad, abogacía, medicina, etc. Sin duda, que el modelo deberá reformularse para modificar las áreas de formación e inclusive, incluir (o excluir) algún tipo de comparación que sea más acorde a los planes de estudios de la(s) carrera(s) que se analizan.

\subsection{Discusión general}

La globalización está cambiando dramáticamente las prácticas profesionales, con una mayor movilidad laboral, los profesionales, en particular, los ingenieros tienen la necesidad y obligación de relacionarse con personas de variadas disciplinas, e inclusive de distintas culturas. Es común desenvolverse en equipos de trabajos "dispersos" (en distintas zonas geográficas), multidisciplinarios y multiculturales (Lloyd John).

Junto con el efecto globalización sobre la práctica profesional del ingeniero, se aprecia un fuerte incremento de profesionales ingenieros en Chile, además de la enorme generación de ingenieros 
procedentes de China e India, conduce prontamente a una comoditización de las competencias en ingeniería.

Ante escenario descrito, es necesario obtener una fuente de ventaja competitiva, la cual puede provenir de la capacidad de los profesionales de liderar y trabajar con equipos dispersos, multiculturales y multidisciplinarios.

En los currículos de ingeniería nacionales analizados, no existen instancias formales de adquisición de competencias en este ámbito. Es posible que existan iniciativas esporádicas en que se produzcan actividades que convoquen a estudiantes de distintas disciplinas, diferentes culturas y dispersos en proyectos comunes.

A través de los párrafos anteriores, se deja la preocupación latente en cuanto a la importancia y creencia de que un currículo de ingeniería pueda adoptar un modelo multidisciplinario (que conlleva a una buena dosis en su flexibilidad), disperso y multicultural a través de la tecnología en la educación del ingeniero del futuro.

Finalmente, considérese que las oportunidades para la ingeniería en el futuro de corto y mediano plazo estarán concentradas en las actividades orientadas al servicio (Hira Ron, 2004). Debido al incremento en la demanda del sector de los servicios, se requerirán ingenieros con destrezas interpersonales, habilidad para trabajar en equipos (que incluyan profesionales no ingenieros) y tener amplios conocimientos de tecnología, de la sociedad y de los negocios. Considerando lo anterior, será muy conveniente considerar implementar asignaturas que incentiven dichas habilidades mencionadas, o se corre el riesgo de perder competitividad.

En el contexto nacional chileno, será necesario incorporar al currículum componentes formales orientadas al emprendimiento en los cuales se desarrolle o se potencien en los estudiantes habilidades de tipo perceptivas como la curiosidad, apretura de mente y flexibilidad. Son cualidades necesarias para lograr incentivar en los ingenieros la creación de nuevas empresas que impulsen el desarrollo del país.

\section{BIBLIOGRAFÍA}

BOGAN, CHRISTOPHER, Benchmarking Best Prectice, McGraw Hill, 1994

BOXWELL, ROBERT, Benchmarking for a Competitive Adventage, MCGraw Hill, 1994

SteVenson, WILLIAM, Productions/Operations Management, Irwin Publishing Company, $5^{\text {ta }}$ Edición, 1996.

CÁceres, GuStAVo, Algunos Objetivos Estratégicos para el Mejoramiento de la Educación Técnica Superior, (Diciembre 2002). Boletín número 3 MECESUP del Ministerio de Educación de Chile, 1525.

HIRA, RON, Undergraduate Engineering Education Curriculum and Educational Research, IEEE International Symposium on Technology and Society. Extraído en Noviembre 2004 de http://www.pac.gmu.edu/

LLOYD JOHN R., HINDS TIMOTHY J., DAVID KENNETH, CHUNG JUNG MOOON, INTEnD: A Dispersered Desing Team Approach for the Globalization of Engineering Education. Extraído de http://www.asme.org/education/enged/awards. 
Tryggvason Gretar, Thouless Michael, Dutta Deba, Ceccio L. Steven, Tilbury M. Dawn,, The New Mechanical Engineering Curriculum at the University of Michigan (Julio 2001). Journal of Engineering Education, 437-444.

CNAP, Comisión Nacional de Acreditación de Pregrado, Manual para el desarrollo de procesos de autoevaluación. Extraído en Marzo 2003 de http://www.cnap.cl.

CNAP, Comisión Nacional de Acreditación de Pregrado, Información de opinión en procesos de autoevaluación técnicas e instrumentos (APE). Extraído en Marzo 2003 de http://www.cnap.cl.

Pontificia Universidad Católica de Chile, Plan de estudio de Ingeniería Civil Electricista, documento en línea extraído en Octubre 2004 de http://www2.ing.puc.cl/dirdoc/index.phtml?url=info_academica/curriculo/curriculo98/titulos_grados/ci v_ele.phtml

Pontificia Universidad Católica de Valparaíso, Plan de estudio de Ingeniería Civil Electrónica, documento en línea extraído en Octubre 2004 de http://www.ucv.cl/p3_carrera/site/pags/20031229161238.html

Universidad De ChILE, Plan de estudio de Ingeniería Civil Electricista, documento en línea extraído en Octubre 2004 de http://www.die.uchile.cl/

Universidad de Santiago de Chile, Plan de estudio de Ingeniería Civil en Electricidad, documento en línea, extraído en Octubre 2004 de http://ingelec.usach.cl/

Universidad TÉcnica Federico Santa María, Plan de estudio de Ingeniería Civil Electrónica, documento en $\begin{array}{lllll}\text { línea extraído } & \text { en } & \text { Octubre } & 2004 & \text { de }\end{array}$ http://www.utfsm.cl/academia/carreras/pregrado/6anos/ing.civil.electronica.htm. 


\title{
Contactar
}

Revista lberoamericana de Educación

\author{
Principal OEI
}

